\title{
Transcranial direct current stimulation for spinal cord injury- associated neuropathic pain
}

\author{
Caixia $\mathrm{Li}^{1}$, Sukunya Jirachaipitak ${ }^{2}$, Paul Wrigley ${ }^{3,4},{\text { Hua } \mathrm{Xu}^{1} \text {, and Pramote Euasobhon }}^{2}$ \\ 'Department of Anesthesiology, Yueyang Hospital of Integrated Traditional Chinese and Western Medicine, Shanghai University of Traditional \\ Chinese Medicine, Shanghai, China \\ ${ }^{2}$ Department of Anesthesiology, Faculty of Medicine Siriraj Hospital, Mahidol University, Bangkok, Thailand \\ ${ }^{3}$ Pain Management Research Institute, Faculty of Medicine and Health, Northern Clinical School, The University of Sydney, Sydney, Australia \\ ${ }^{4}$ Kolling Institute, Northern Sydney Local Health District and The University of Sydney at Royal North Shore Hospital, Sydney, Australia
}

Received October 26, 2020

Revised December 26, 2020

Accepted December 30, 2020

Handling Editor: Kyung Hoon Kim

\section{Correspondence}

Sukunya Jirachaipitak

Department of Anesthesiology,

Faculty of Medicine Siriraj Hospital,

Mahidol University, 2 Wanglang Road,

Bangkoknoi, Bangkok 10700, Thailand

Tel: +66-2-419-7995

Fax: +66-2-411-3256

E-mail: kobemed56@hotmail.com
Several types of pain occur following spinal cord injury (SCI); however, neuropathic pain (NP) is one of the most intractable. Invasive and non-invasive brain stimulation techniques have been studied in clinical trials to treat chronic NP following SCI. The evidence for invasive stimulation including motor cortex and deep brain stimulation via the use of implanted electrodes to reduce SCl-related NP remains limited, due to the small scale of existing studies. The lower risk of complications associated with non-invasive stimulation, including transcranial direct current stimulation (tDCS) and repetitive transcranial magnetic stimulation (rTMS), provide potentially attractive alternative central neuromodulation techniques. Compared to rTMS, tDCS is technically easier to apply, more affordable, available, and potentially feasible for home use. Accordingly, several new studies have investigated the efficacy of tDCS to treat NP after SCI. In this review, articles relating to the mechanisms, clinical efficacy and safety of tDCS on SCI-related NP were searched from inception to December 2019. Six clinical trials, including five randomized placebo-controlled trials and one prospective controlled trial, were included for evidence specific to the efficacy of tDCS for treating SCl-related NP. The mechanisms of action of tDCS are complex and not fully understood. Several factors including stimulation parameters and individual patient characteristics may affect the efficacy of tDCS intervention. Current evidence to support the efficacy of utilizing tDCS for relieving chronic NP after SCI remains limited. Further strong evidence is needed to confirm the efficacy of tDCS intervention for treating SCl-related NP.

Key Words: Chronic Pain; Deep Brain Stimulation; Electric Stimulation Therapy; Electrodes, Implanted; Motor Cortex; Neuralgia; Spinal Cord Injuries; Transcranial Direct Current Stimulation; Transcranial Magnetic Stimulation; Treatment Outcome.

\section{INTRODUCTION}

Spinal cord injury (SCI) is a catastrophic condition that results in damage to or loss of autonomic, sensory, and motor functions that can lead to multiple types of secondary complications. Pain is a very common secondary condition among SCI patients, and its impact on patient quality of life and clinical outcomes is profound [1]. Although several types of pain occur following SCI, neuropathic pain (NP) which occurs in almost one half of people with SCI (c) This is an open-access article distributed under the terms of the Creative Commons Attribution Non-Commercial License (http://creativecommons.org/licenses/by-nc/4.0/), which permits unrestricted non-commercial use, distribution, and reproduction in any medium, provided the original work is properly cited.

(C) The Korean Pain Society, 2021
Author contributions: Caixia Li: Writing/manuscript preparation; Sukunya Jirachaipitak: Project administration; Paul Wrigley: Writing/manuscript preparation; Hua Xu: Supervision; Pramote Euasobhon: Study conception. 
is most commonly rated as severe [2]. Despite the fact that pregabalin and gabapentin have been demonstrated an effective treatment in reducing SCI-related NP [3], some patients still have refractory NP to currently available standard treatments and face the challenge of managing lifelong severe chronic pain $[4,5]$.

The high level of treatment failure in this pain setting has precipitated increased interest in alternative neuromodulatory stimulation approaches. Different types of invasive and non-invasive brain stimulation have been proposed and investigated to treat SCI-related pain. Deep brain stimulation can be performed via implantation of intracranial electrodes [6], however, its uncertain efficacy and the high risk associated with this invasive procedure has made this technique uncommon and increased interest in less invasive techniques for brain stimulation [7].

Transcranial direct current stimulation (tDCS) is a noninvasive clinical tool developed to modulate brain activity via the use of large electrodes that are attached to the scalp, over the area of the brain to be targeted, to deliver a mild continuous current [8]. Clinical trials involving tDCS have developed over the years to include the management of pain. Early studies in humans investigated the use of tDCS to treat or modify psychiatric disorders, most notably depression [9].

tDCS was later introduced as a non-invasive technique for modifying cortical activity in humans to alter behavioral, perceptual, and cognitive functions [10], as well as memory and learning. tDCS has been proposed for use in many conditions including Parkinson's disease, neuropsychiatric disorders, stroke, migraine headache, and drug addiction [11-14], as well as chronic and acute pain [8]. A small number of clinical studies have investigated the use of tDCS to treat patients with chronic pain associated with SCI. A landmark positive sham-controlled study using tDCS in SCI-related NP [15] generated significant interest given the continued challenge of managing this type of pain despite all available treatment options and advancement in SCI medicine.

The aim of this review was to present what is currently known about the evidence specific to the efficacy of tDCS, the potential mechanisms of action of tDCS, and the factors that influence its efficacy for treating SCI-related NP.

\section{MAIN BODY}

\section{Literature search strategy}

Titles and abstracts were identified in the MEDLINE, PubMed, and EMBASE databases from inception to December 2019. The search terms combined the follow- ing keywords: transcranial direct current stimulation, tDCS, transcranial DC stimulation, non-invasive brain stimulation, motor cortex stimulation, cerebral cortical stimulation, transcranial electrical stimulation, neurostimulation, neuromodulation, spinal cord injury, and neuropathic pain. Articles relating to the mechanisms, clinical efficacy, and safety of tDCS on SCI-related NP were eligible for inclusion. The references of retrieved articles were also hand searched to find additional articles that may have been missed.

Experimental studies regarding animals or healthy subjects were also included if they were relevant to the mechanisms of action and influential factors determining the effects of tDCS relevant to SCI pain. Non-English articles and studies on patients with primary symptoms other than pain were excluded. Original clinical trials were screened for evidence specific to the efficacy of tDCS for treating SCI-related NP and six placebo-controlled trials including five randomized controlled trials and one prospective controlled trial were found (Table 1).

\section{Evidence specific to the efficacy of tDCS for treating SCI-related NP}

According to the basic principles of pathophysiology, any treatment strategy that can reverse physiological, biochemical, and anatomical changes at the spinal and supraspinal levels may be of benefit for improvement of a pain condition. The observed effects of tDCS on cortical excitability led to trials to investigate the efficacy of tDCS via its potential central modulation effects for reducing $\mathrm{NP}$ following SCI.

Most studies assessing the efficacy of tDCS for NP after SCI apply anodal stimulation to the primary motor cortex (M1) of the hemisphere contralateral to the pain, with cathode placement over the supraorbital region ipsilateral to the pain. Stimulation at $2 \mathrm{~mA}$ intensity using an electrode of $35 \mathrm{~cm}^{2}$ in size was delivered for 20 minutes per day and repeated for 5 to 10 sessions in the majority of studies [15-17]. The efficacy of a single session of tDCS was also investigated in a randomized, double-blind, crossover study [18].

The original study by Fregni et al. [15] found that patients who received active tDCS ( $2 \mathrm{~mA}$ for $20 \mathrm{~min}$ for five consecutive days) experienced a significant (approximately $\geq 50 \%$ ) reduction in pain which was not observed after sham stimulation. A significant cumulative analgesic effect was observed with the peak pain reduction reached after the last stimulation session. Patients also had less pain compared to baseline in the active tDCS group for two weeks after the last session of stimulation. Moreover, they found a strong negative relationship between pain 


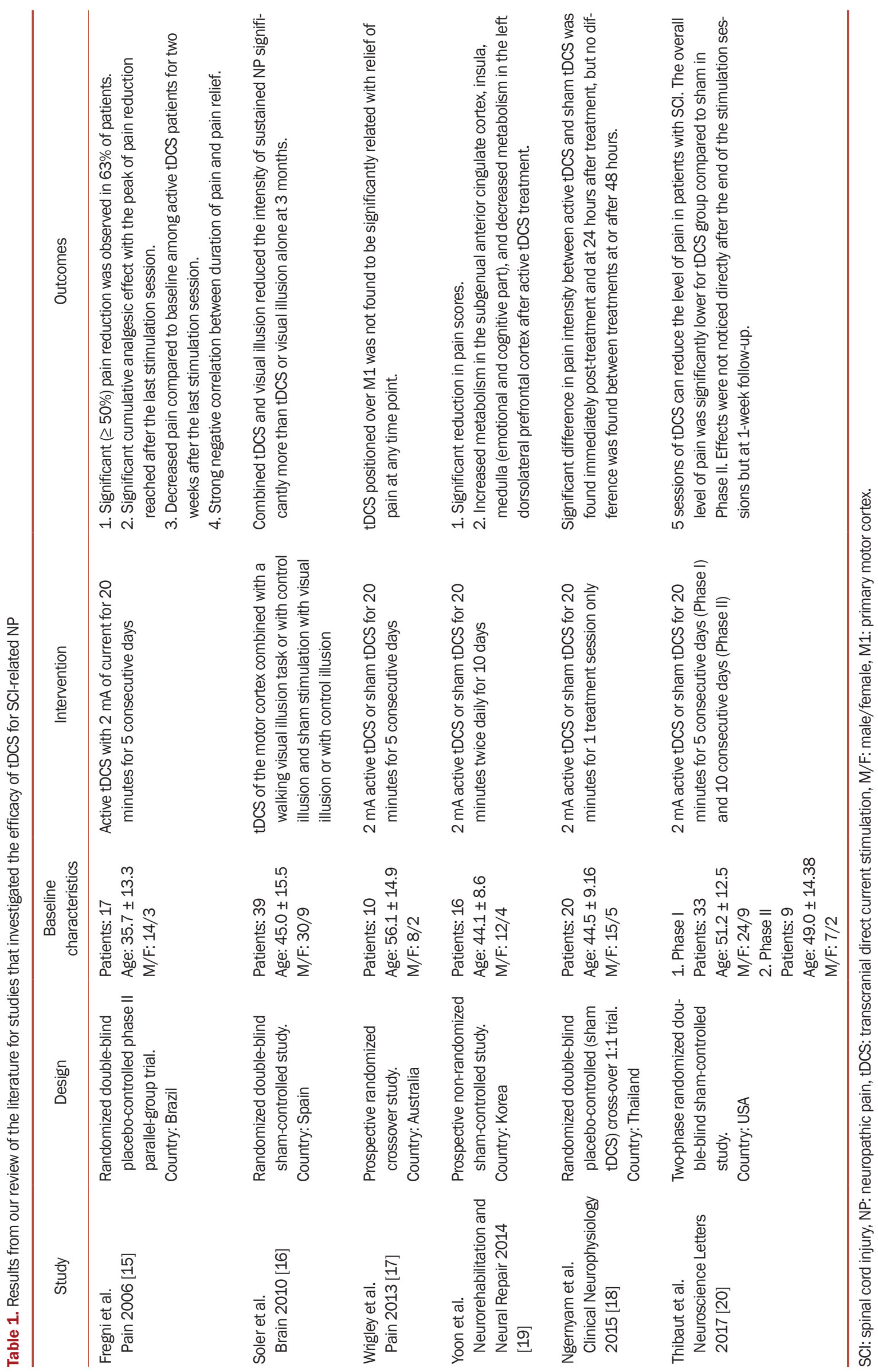


duration and pain relief, suggesting patients with shorter disease duration had greater pain reduction.

Yoon et al. [19] found a statistically significant reduction in pain scores after active tDCS treatment involving 20 sessions within a 2-week period for patients with NP after SCI. However, meaningful clinical improvement was observed in only $2(20 \%)$ patients, with a decrease in pain intensity from baseline of greater than $30 \%$. The results of Yoon et al. [19] were less efficacious than Fregni et al. [15] who observed a decrease of equal to or greater than $50 \%$ in the visual analog scale (VAS) for overall pain in more than half (63\%) of patients with NP following SCI. To explain this difference Yoon et al. [19] suggested that evaluation of only overall pain (and not specific types of pain, such as paroxysmal or continuous pain) in their study could have influenced the observed higher rate of pain improvement. The pain mitigation effect of tDCS has been reported to vary among pain subtypes, including spontaneous pain, paroxysmal pain, and continuous pain [16].

A randomized double-blind sham-controlled trial performed by Soler et al. [16] examined the analgesic effect of tDCS of the motor cortex combined with a walking visual illusion task. That study included thirty-nine patients randomized into four different groups involving: tDCS with walking visual illusion, tDCS with control illusion and sham stimulation, tDCS with visual illusion or tDCS with control illusion. They observed that combined tDCS and visual illusion significantly reduced the intensity of NP when compared with tDCS or visual illusion alone. The combined group demonstrated sustained pain improvement at 3 months, whereas no improvement was observed in the other groups. Visual illusion can influence corticospinal excitability, so some synergy has been proposed to occur when visual illusion and tDCS are combined. These findings raise the possibility that when tDCS is combined with other therapeutic modalities, an enhancement of indirect stimulation effect may occur [16].

An addition randomized double-blind controlled crossover study by Ngernyam et al. [18] using a single session of $2 \mathrm{~mA}$ active or sham tDCS treatment for 20 minutes found a significant difference in pain intensity between sham and active tDCS immediately and at 24 hours after treatment. However, there was no difference in pain intensity between groups at 48 hours [18].

A recent randomized sham-controlled clinical trial conducted by Thibaut et al. [20] reported delayed NP reduction following M1 tDCS in patients with SCI. In this study 33 patients were enrolled in a two-phase tDCS treatment, with Phase I consisting of 5 days tDCS with a review at 3 months, followed by Phase II involving 10 days of tDCS with review at 2 months. The results showed a treatment effect at 1-week follow-up for Phase I and at 4-week follow- up for Phase II. However, the effects were not observed immediately after the end of the tDCS treatment sessions. The sham group had a higher area under curve for average VAS score $(P=0.026)$ and VAS least $(P=0.011)$, demonstrating that the overall level of pain was significantly lower for the tDCS group compared to the sham group in Phase II [20].

However, not all studies have demonstrated beneficial effects from tDCS for SCI-related NP. Wrigley et al. [17] investigated the efficacy of tDCS in patients with SCI-related NP in a randomised blinded sham-controlled crossover study. They evaluated the immediate and longer-term effects of 5 daily sessions of tDCS on pain, unpleasantness, and mood up to 6 months. They found tDCS positioned over Ml was not associated with pain relief at any time point. When compared to the subjects in Fregni et al. [15] the average injury duration was much longer. Wrigley et al. [17] raised the possibility that once consolidation of central changes caused by an SCI occurs, tDCS may be no longer able to modulate central pain-related circuits, however, further research was needed to establish this [17]. Of note, in a follow-up study (Soler et al. [16], discussed above) by the same group involved with the Fregni et al. [15] paper tDCS alone did not result in a significant reduction in ongoing pain except when combined with visual imagery.

Overall, the scientific evidence relating to the efficacy of tDCS for treating NP after SCI is limited and conflicting, limiting our ability to reach definitive conclusions. Many factors including patient factors (e.g., SCI duration, variability of SCI types, central NP subtypes), technical factors in tDCS delivery (e.g., current density and direction, intensity and duration of stimulation, number of sessions) and study design (e.g., blinding, randomization, and subject numbers) influence the assessment of tDCS efficacy. A European expert panel recently issued a Level C (possible efficacy) recommendation for anodal tDCS of the left M1 (or contralateral to pain side with a right orbitofrontal cathode) in patients with chronic NP of the lower limbs secondary to SCI [21].

\section{Mechanisms of action of tDCS}

The potential analgesic mechanisms involved with tDCS have not been conclusively established; however, several mechanisms have been suggested. The most widely proposed mechanism is that tDCS modulates spontaneous cortical neuronal activity by polarizing the resting membrane which may last up to 90 minutes [22]. tDCS does not trigger action potentials but affects the spike timing of individual neurons receiving suprathreshold inputs [23]. The effects of tDCS on cortical excitability are polarity-dependent. The anode depolarizes the neuronal membrane 
and increases cortical excitability whereas the cathode hyperpolarizes the neuronal membrane and decreases cortical excitability [24]. Anodal tDCS of the motor cortex, resulting in activation of thalamic and subthalamic nuclei, influences several pain-related structures such as the anterior cingulate, the periaqueductal gray, and eventually modulates both pain perception and the affectiveemotional component of the pain experience [25]. Severalminute stimulation of the transcranial electric current results in a polarity-dependent induction of glutamatergic calcium-dependent neuroplasticity, which are thought to be the result of modification of N-methyl-D-aspartate (NMDA) receptor activation $[26,27]$.

NMDA receptors have been widely investigated regarding their function in excitatory synaptic transmission during chronic pain development and induction of synaptic plasticity leading to a modification of synaptic strength [28]. Suppression of both cathodal and anodal after-effects by the NMDA-receptor antagonist dextromethorphan, suggested that the induction of synaptic plasticity rather than glutamatergic excitatory signal transmission may represent the relevant contribution of NMDA receptors to the after-effects of tDCS [24]. Moreover, the modulating effect of the partial NMDA receptor agonist, d-cycloserine, increases anodal tDCS-induced excitability and enhances the after-effects of stimulation [29]. Therefore, anodal tDCS probably induces neuroplasticity by increasing cortical NMDA-receptor activity, which is a dependent mechanism for the after-effects of tDCS.

Moreover, administration of lorazepam, which is a gamma-aminobutyric acid $A\left(G_{A B A_{A}}\right)$ receptor agonist, can cause early-inhibition and late-enhancement of tDCS-induced plasticity [30]. Also, the measurement of changes in neurotransmitter concentrations by magnetic resonance spectroscopy suggested that anodal tDCS significantly decreased cortical GABAergic activity [31]. Other neurochemical changes in the cortex have also been proposed during anodal tDCS with increased cortical myoinositol beneath the stimulating electrode. Myoinositol, as an essential component of the phosphoinositide pathway, can influence the basis of neuronal signal transmission and brain plasticity. However, no change in another neuronal marker, $\mathrm{N}$-acetyl-aspartate, was observed in this study [32].

Other mechanisms potentially involved in the effect of tDCS have been suggested. For example, tDCS altered spontaneous neuronal oscillations by increasing slow electroencephalogram (EEG) activities [33]. Several studies have employed positron emission tomography (PET) or functional magnetic resonance imaging (fMRI) to assess tDCS-influenced changes in brain activity. A study in healthy subjects using PET found that tDCS resulted in sustained and disseminated changes in regional neuronal activity and regional cerebral blood flow [34]. DosSantos et al. [35] used carfentanil, a $\mu$-opioid receptor selective radiotracer, in a PET study to visualize the availability of $\mu$-opioid receptors during tDCS in a subject with chronic pain of the temporomandibular joint. They found that a single active tDCS session decreased $\mu$-opioid receptor non-displaceable binding potential levels in subcortical pain matrix structures compared to a sham, including the anterior cingulate cortex, nucleus accumbens, insula, and most notably in the posterior thalamus, all of which suggests that the reduced pain experienced during and after tDCS may be due to enhanced endogenous opioid release [35].

Another study using PET with 18F-fluorodeoxyglucose to evaluate the effect of tDCS on pain found an increased metabolism in the insula, subgenual anterior cingulate cortex, and medulla [19]. It also reported a reduced metabolism in the left dorsolateral prefrontal cortex during tDCS when compared with a sham. Changes in these areas were felt relevant to the pain mitigation effects of tDCS.

Changes in a number of neurotransmitters in the central nervous system have been shown to alter the effect of tDCS. Serotoninergic activation by citalopram, a serotonin reuptake inhibitor, has been shown to enhance and prolong the neuroplastic excitability accomplished by anodal tDCS, known as anodal tDCS-induced facilitatory plasticity, and to convert cathodal tDCS-induced inhibitory plasticity into facilitation [36]. In contrast, administration of Ldopa transforms anodal tDCS-induced cortical excitability into inhibition, and prolongs the diminution of cathodal tDCS-induced excitability [37]. A recent experimental NP study conducted in an animal model was the first to report the involvement of the cannabinoid receptors 1 and 2 (CB1 and CB2) in tDCS-induced pain reduction [38].

Taken together, the aforementioned findings suggest that tDCS influences several physiological processes in the central nervous system and the peripheral nervous system (Fig. 1) that may be responsible for its effects. However, a more definitive understanding of the mechanism of action of tDCS is needed to unlock the true potential of tDCS for treating pain [39].

\section{Factors determining the efficacy of tDCS}

Several factors, including current density, stimulation duration, and current direction can influence the efficacy of tDCS [11].

Current density, which is determined by current intensity and electrode size, is the factor that most greatly affects the degree of cortical excitability [40]. The greater the current density that is delivered, the greater the effect of tDCS. However, increases in current density can increase 


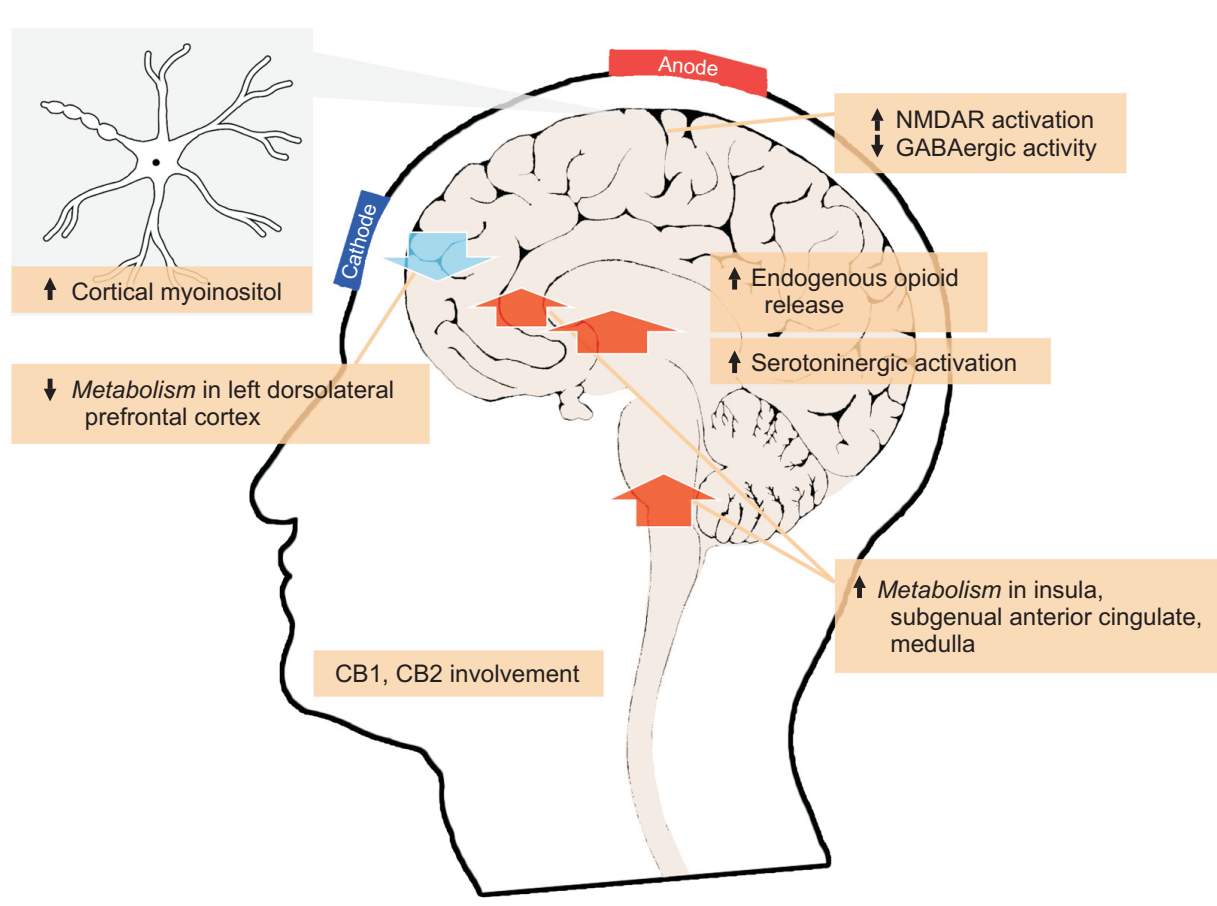

Fig. 1. Summary of neurophysiological mechanisms of transcranial direct current stimulation. CB1: cannabinoid receptor 1, CB2: cannabinoid receptor 2, GABA: gamma-aminobutyric acid, NMDAR: $\mathrm{N}$ methyl-D-aspartate receptor. cutaneous pain and may affect more deeply located cortical neurons.

Stimulation duration was reported to be a factor that influenced the duration of after-effects following tDCS [22]. Moreover, it has been suggested that daily tDCS sessions are needed to achieve continuation of after-effects for days or weeks.

The orientation of the electric field is determined by the size, position, and polarity of the electrode. It is necessary to correctly position the electrodes to facilitate current flow from anode to cathode. Anodal electrode stimulation generally enhances motor cortex excitability, whereas cathodal electrode stimulation yields the opposite effect. Uncontrolled interference with ongoing cortical activity during tDCS should be avoided. tDCS-induced excitability is highly dependent on the state of the stimulated motor cortex of the patient during stimulation. Massive motor exercise can nullify the cortical excitability effects of tDCS [41].

The conductivity of the electrical current can be enhanced by swabbing the skin and scalp with alcohol to remove oil and debris. This will result in reduced impedance and improved homogeneity of the electric field under the electrodes. The electrode sponge should be moistened with water, sodium chloride solution, or electrode gel to reduce the resistance and enhance current flow.

Other factors, including the presence of depressionrelated symptoms, duration of pain, and concurrent drug therapy, may influence the ability of the tDCS effect. A recent systematic review and meta-analysis found that studies that excluded depressed patients observed signifi- cantly better tDCS-related pain relief; however, studies that enrolled patients with depressive symptoms did not observe a similar significant effect. Moreover, studies that included subjects with a duration of NP of less than 5 years demonstrated a higher likelihood of reporting a decrease in pain intensity compared to studies that enrolled patients with a duration of pain lasting longer than 5 years [42]. It has also been reported that several classes of medications, including calcium and sodium channel blockers, and agents that impact various neurotransmitter systems (e.g., dopamine, GABA, and serotonin) may influence tDCS after-effects [43].

Even with strict adherence to the treatment protocol, outcomes can vary significantly among patients. Factors that have been proposed to explain these different outcomes include patient age [44] and the time of day that the treatment was administered [45]. Genetic factors are also likely to significantly influence treatment. Brain-derived neurotrophic factor gene (BDNF) polymorphism has been proposed to play a significant role in a patient's susceptibility to plasticity induced by tDCS [46]. However, the inter-individual differences still need to be investigated and understood, similar to most treatment modalities in pain management.

\section{Adverse effects of tDCS}

One of the appealing characteristics of tDCS is its noninvasive nature. Moreover, almost all of the side effects of tDCS are transient and well-tolerated. The possible side effects of tDCS include nausea, headache, discomfort, 
dizziness, itching, pain, tingling, blurred vision, burning sensation, fatigue, and skin irritation under the electrodes [47]. A recent review found that the most common adverse effects of tDCS for chronic pain during or after the tDCS protocol, included primarily tingling at the site of stimulation (active tDCS - 44\%, sham tDCS - 47\%) and fatigue or sleepiness (after active tDCS - 31\%, after sham tDCS - 21\%) [48]. One pain therapy study observed electrode-induced skin burn in 3 patients; however, all burns healed within a few days, but one patient was left with a small scar [49]. Accordingly, patients should be interviewed and evaluated for the presence of skin diseases and conditions of the skin under the electrodes before and after tDCS. Mild redness is commonly observed under the electrodes following treatment; however, this mild discoloration rapidly fades.

Patients with pacemakers or other electrical/metallic implants located near the electrodes are normally contraindicated for tDCS. Though the incidence of seizure has not been shown to increase in normal healthy subjects, there is no data in patients with epilepsy. As a result, patients with a history of seizure should not be treated with tDCS. Although the majority of the adverse effects that have been reported are relatively minor, most of those studies used tDCS with low current intensity $(\leq 2 \mathrm{~mA})$ [11]. Therefore, future studies that employ tDCS with greater levels of electrical current intensity should carefully monitor their patients for additional adverse effects.

\section{CONCLUSIONS}

tDCS is a well-tolerated, non-invasive, and potentially easy-to-use non-invasive brain stimulation modality that has been proposed as a treatment option in the management of pain. However, evidence supporting the efficacy of tDCS for relief of chronic NP after SCI remains limited. Moreover, the mechanisms of action of tDCS are complex and not fully understood.

Additional and stronger evidence is needed to confirm the efficacy and duration of the effect of tDCS for treating SCI-related NP. This may be assisted by the availability of home-based tDCS units however the safety of these devices would need to be carefully monitored. Consensus on the optimal trial protocol to limit the variability of stimulation parameters would enable better comparison between trials and an increasing in the strength of the evidence.

\section{ACKNOWLEDGMENTS}

The authors gratefully acknowledge Mr. Virachat Sanan- silp for figure creation and Miss Nattaya Bunwatsana for administrative support.

\section{CONFLICT OF INTEREST}

No potential conflict of interest relevant to this article was reported.

\section{FUNDING}

No funding to declare.

\section{ORCID}

Caixia Li, https://orcid.org/0000-0001-6330-1001

Sukunya Jirachaipitak, https://orcid.org/0000-0001-5188-3286

Paul Wrigley, https://orcid.org/0000-0003-0960-4102

Hua Xu, https://orcid.org/0000-0001-5607-7742

Pramote Euasobhon, https://orcid.org/0000-0001-5268-5476

\section{REFERENCES}

1. Hatch MN, Cushing TR, Carlson GD, Chang EY. Neuropathic pain and SCI: identification and treatment strategies in the 21st century. J Neurol Sci 2018; 384: 75-83.

2. Siddall PJ, Taylor DA, McClelland JM, Rutkowski SB, Cousins MJ. Pain report and the relationship of pain to physical factors in the first 6 months following spinal cord injury. Pain 1999; 81: 187-97.

3. Davari M, Amani B, Amani B, Khanijahani A, Akbarzadeh A, Shabestan R. Pregabalin and gabapentin in neuropathic pain management after spinal cord injury: a systematic review and meta-analysis. Korean J Pain 2020; 33: 3-12.

4. Widerström-Noga EG, Duncan R, Felipe-Cuervo E, Turk DC. Assessment of the impact of pain and impairments associated with spinal cord injuries. Arch Phys Med Rehabil 2002; 83: 395-404.

5. Finnerup NB, Yezierski RP, Sang CN, Burchiel KJ, Jensen TS. Treatment of spinal cord injury pain. Pain Clin Updates 2001; 9: 1-6.

6. Wallace BA, Ashkan K, Benabid AL. Deep brain stimulation for the treatment of chronic, intractable pain. Neurosurg Clin N Am 2004; 15: 343-57.

7. Chari A, Hentall ID, Papadopoulos MC, Pereira EA. Surgical neurostimulation for spinal cord injury. Brain Sci 2017; 7: 18.

8. Lefaucheur JP, Antal A, Ahdab R, Ciampi de Andrade D, Fregni F, Khedr EM, et al. The use of repetitive transcranial magnetic stimulation (rTMS) and transcranial direct current 
stimulation (tDCS) to relieve pain. Brain Stimul 2008; 1: 33744.

9. Costain R, Redfearn JW, Lippold OC. A controlled trial of the therapeutic effect of polarization of the brain in depressive illness. Br J Psychiatry 1964; 110: 786-99.

10. Miniussi C, Cappa SF, Cohen LG, Floel A, Fregni F, Nitsche MA, et al. Efficacy of repetitive transcranial magnetic stimulation/transcranial direct current stimulation in cognitive neurorehabilitation. Brain Stimul 2008; 1: 326-36.

11. Nitsche MA, Cohen LG, Wassermann EM, Priori A, Lang N, Antal A, et al. Transcranial direct current stimulation: state of the art 2008. Brain Stimul 2008; 1: 206-23.

12. Fregni F, Pascual-Leone A. Technology insight: noninvasive brain stimulation in neurology-perspectives on the therapeutic potential of rTMS and tDCS. Nat Clin Pract Neurol 2007; 3: 383-93.

13. George MS, Aston-Jones G. Noninvasive techniques for probing neurocircuitry and treating illness: vagus nerve stimulation (VNS), transcranial magnetic stimulation (TMS) and transcranial direct current stimulation (tDCS). Neuropsychopharmacology 2010; 35: 301-16.

14. Fregni F, Nitsche MA, Loo CK, Brunoni AR, Marangolo P, Leite J, et al. Regulatory considerations for the clinical and research use of transcranial direct current stimulation (tDCS): review and recommendations from an expert panel. Clin Res Regul Aff 2015; 32: 22-35.

15. Fregni F, Boggio PS, Lima MC, Ferreira MJ, Wagner T, Rigonatti SP, et al. A sham-controlled, phase II trial of transcranial direct current stimulation for the treatment of central pain in traumatic spinal cord injury. Pain 2006; 122: 197-209.

16. Soler MD, Kumru H, Pelayo R, Vidal J, Tormos JM, Fregni F, et al. Effectiveness of transcranial direct current stimulation and visual illusion on neuropathic pain in spinal cord injury. Brain 2010; 133: 2565-77.

17. Wrigley PJ, Gustin SM, McIndoe LN, Chakiath RJ, Henderson LA, Siddall PJ. Longstanding neuropathic pain after spinal cord injury is refractory to transcranial direct current stimulation: a randomized controlled trial. Pain 2013; 154: 217884.

18. Ngernyam N, Jensen MP, Arayawichanon $\mathrm{P}$, Auvichayapat $\mathrm{N}$, Tiamkao S, Janjarasjitt $S$, et al. The effects of transcranial direct current stimulation in patients with neuropathic pain from spinal cord injury. Clin Neurophysiol 2015; 126: 382-90.

19. Yoon EJ, Kim YK, Kim HR, Kim SE, Lee Y, Shin HI. Transcranial direct current stimulation to lessen neuropathic pain after spinal cord injury: a mechanistic PET study. Neurorehabil Neural Repair 2014; 28: 250-9.

20. Thibaut A, Carvalho S, Morse LR, Zafonte R, Fregni F. Delayed pain decrease following M1 tDCS in spinal cord injury: a randomized controlled clinical trial. Neurosci Lett 2017; 658: 19-26.

21. Lefaucheur JP, Antal A, Ayache SS, Benninger DH, Brunelin
J, Cogiamanian F, et al. Evidence-based guidelines on the therapeutic use of transcranial direct current stimulation (tDCS). Clin Neurophysiol 2017; 128: 56-92.

22. Nitsche MA, Paulus W. Sustained excitability elevations induced by transcranial DC motor cortex stimulation in humans. Neurology 2001; 57: 1899-901.

23. Anastassiou CA, Perin R, Markram H, Koch C. Ephaptic coupling of cortical neurons. Nat Neurosci 2011; 14: 217-23.

24. Nitsche MA, Müller-Dahlhaus F, Paulus W, Ziemann U. The pharmacology of neuroplasticity induced by non-invasive brain stimulation: building models for the clinical use of CNS active drugs. J Physiol 2012; 590: 4641-62.

25. Knotkova H, Nitsche MA, Cruciani RA. Putative physiological mechanisms underlying tDCS analgesic effects. Front Hum Neurosci 2013; 7: 628.

26. Nitsche MA, Fricke K, Henschke U, Schlitterlau A, Liebetanz $\mathrm{D}$, Lang N, et al. Pharmacological modulation of cortical excitability shifts induced by transcranial direct current stimulation in humans. J Physiol 2003; 553(Pt 1): 293-301.

27. Liebetanz D, Nitsche MA, Tergau F, Paulus W. Pharmacological approach to the mechanisms of transcranial DCstimulation-induced after-effects of human motor cortex excitability. Brain 2002; 125(Pt 10): 2238-47.

28. D'Angelo E, Rossi P. Integrated regulation of signal coding and plasticity by NMDA receptors at a central synapse. Neural Plast 1998; 6: 8-16.

29. Nitsche MA, Jaussi W, Liebetanz D, Lang N, Tergau F, Paulus W. Consolidation of human motor cortical neuroplasticity by D-cycloserine. Neuropsychopharmacology 2004; 29: 1573-8.

30. Nitsche MA, Liebetanz D, Schlitterlau A, Henschke U, Fricke K, Frommann K, et al. GABAergic modulation of DC stimulation-induced motor cortex excitability shifts in humans. Eur J Neurosci 2004; 19: 2720-6.

31. Stagg CJ, Best JG, Stephenson MC, O'Shea J, Wylezinska M, Kincses ZT, et al. Polarity-sensitive modulation of cortical neurotransmitters by transcranial stimulation. J Neurosci 2009; 29: 5202-6.

32. Rango M, Cogiamanian F, Marceglia S, Barberis B, Arighi A, Biondetti $\mathrm{P}$, et al. Myoinositol content in the human brain is modified by transcranial direct current stimulation in a matter of minutes: a 1H-MRS study. Magn Reson Med 2008; 60: 782-9.

33. Ardolino G, Bossi B, Barbieri S, Priori A. Non-synaptic mechanisms underlie the after-effects of cathodal transcutaneous direct current stimulation of the human brain. J Physiol 2005; 568(Pt 2): 653-63.

34. Lang N, Siebner HR, Ward NS, Lee L, Nitsche MA, Paulus W, et al. How does transcranial DC stimulation of the primary motor cortex alter regional neuronal activity in the human brain? Eur J Neurosci 2005; 22: 495-504.

35. DosSantos MF, Love TM, Martikainen IK, Nascimento TD, Fregni F, Cummiford C, et al. Immediate effects of tDCS on 
the $\mu$-opioid system of a chronic pain patient. Front Psychiatry 2012; 3: 93.

36. Nitsche MA, Kuo MF, Karrasch R, Wächter B, Liebetanz D, Paulus W. Serotonin affects transcranial direct currentinduced neuroplasticity in humans. Biol Psychiatry 2009; 66: 503-8.

37. Kuo MF, Paulus W, Nitsche MA. Boosting focally-induced brain plasticity by dopamine. Cereb Cortex 2008; 18: 648-51.

38. Souza A, Martins DF, Medeiros LF, Nucci-Martins C, Martins TC, Siteneski A, et al. Neurobiological mechanisms of antiallodynic effect of transcranial direct current stimulation (tDCS) in a mice model of neuropathic pain. Brain Res 2018; 1682: 14-23.

39. Pelletier SJ, Cicchetti F. Cellular and molecular mechanisms of action of transcranial direct current stimulation: evidence from in vitro and in vivo models. Int J Neuropsychopharmacol 2014; 18: pyu047.

40. Nitsche MA, Paulus W. Excitability changes induced in the human motor cortex by weak transcranial direct current stimulation. J Physiol 2000; 527(Pt 3): 633-9.

41. Antal A, Terney D, Poreisz C, Paulus W. Towards unravelling task-related modulations of neuroplastic changes induced in the human motor cortex. Eur J Neurosci 2007; 26: 2687-91.

42. Mehta S, McIntyre A, Guy S, Teasell RW, Loh E. Effectiveness of transcranial direct current stimulation for the management of neuropathic pain after spinal cord injury: a metaanalysis. Spinal Cord 2015; 53: 780-5.
43. McLaren ME, Nissim NR, Woods AJ. The effects of medication use in transcranial direct current stimulation: a brief review. Brain Stimul 2018; 11: 52-8.

44. Müller-Dahlhaus JF, Orekhov Y, Liu Y, Ziemann U. Interindividual variability and age-dependency of motor cortical plasticity induced by paired associative stimulation. Exp Brain Res 2008; 187: 467-75.

45. Sale MV, Ridding MC, Nordstrom MA. Cortisol inhibits neuroplasticity induction in human motor cortex. J Neurosci 2008; 28: 8285-93.

46. Cheeran B, Talelli P, Mori F, Koch G, Suppa A, Edwards M, et al. A common polymorphism in the brain-derived neurotrophic factor gene (BDNF) modulates human cortical plasticity and the response to rTMS. J Physiol 2008; 586: 5717-25.

47. Brunoni AR, Amadera J, Berbel B, Volz MS, Rizzerio BG, Fregni F. A systematic review on reporting and assessment of adverse effects associated with transcranial direct current stimulation. Int J Neuropsychopharmacol 2011; 14: 1133-45.

48. Antal A, Alekseichuk I, Bikson M, Brockmöller J, Brunoni AR, Chen $\mathrm{R}$, et al. Low intensity transcranial electric stimulation: safety, ethical, legal regulatory and application guidelines. Clin Neurophysiol 2017; 128: 1774-809.

49. Oliveira LB, Lopes TS, Soares C, Maluf R, Goes BT, Sá KN, et al. Transcranial direct current stimulation and exercises for treatment of chronic temporomandibular disorders: a blind randomised-controlled trial. J Oral Rehabil 2015; 42: 723-32. 